\title{
Select Bibliography of Higher Education in Canada
}

R. Harris, Marcel de Grandpré, Richard Greene*

\section{RECENT PUBLICATIONS ON CANADIAN EDUCATION}

A selected list of approximately 100 titles bearing on post-secondary education in Canada will appear in each issue of Stoa. These bibliographies are intended to provide a continuing supplement to the three volumes which have been published by the University of Toronto Press: A Bibliograpby of Higher Education in Canada (1960), the 1965 Supplement to A Bibliography of Higher Education, and the recently published 1971 Supplement.

The 1971 Supplement tepresented an attempt to provide a complete listing of books, articles, theses and reports which appeared during the six-year period ending December 31, 1969 ; but it also listed items published during the first three months of 1970. The list which follows here is limited to additional books, articles and reports published during the last nine months of 1970 . The next two issues of Ston will contain titles published in 1971.

The items which appear in the Stoa lists are classified in accordance with the categories adopted in the 1971 Supplement. Unless the title is self-explanatory a brief annotation normally will be supplied. (Owing to space limitations in this issue annotations have been omitted.) When no title is included in a given classification the classification itself is omitted. E.g. below 1. B : , 2. A.

* Robin HARRIS : Chairman of the Higher Education Group, University of Toronto.

* Marcel de GRANDPRE: Professeur à la Faculté des sciences de l'éducation, Université de Montréal.

* Richard GREENE: Bibliotbécaire en chef, Association des Universités et Collèges du Canada. 


\section{I. - DEGREE-GRANTING UNIVERSITIES AND COLLEGES}

\section{History and Organization}

C. The Universities of Quebec

Gauthier, G. "Le Conseil des Universités du Québec", Revize AUPELF VIII, (Printemps 1970), 11-13.

Audet, L.P. «Programme et Professeurs du Collège de Québec, 1635-1763 ", Cabiers des Dix, 34 (1969), 13.38.

Benoist, J. "Centre de Recherches Caraïbes de l'Université de Montréal 》, Revue AUPELF VIII, 2 (Automne 1970) 105-08.

Juhasz, A. « Le Système de documentation de l'Institut de Médecine et de Chirurgie Expérimentales de l'Université de Montréal \%, Revue AUPELF VIII, 1 (Printemps 1970), 14-24.

D. The Universities of Ontario

Cragg AW. "The Crisis at Laurentian", Ontatio Confederation of University Fatulty Association Newsletter IV, 2 (November, 1970), 12-13.

Feeley, J. "A Library in Crisis: the University of Toronto Library, 1890-1892," Ontario History, LXII (December, 1970), 220-34.

\section{Current Trends and Problems}

A. General

Blishen, B.R. "Social Class and Opportunity in Canada", Can. Rev. Sociology Antbropology VII, 2 (May, 1970), 110-27.

Carrier H. L'Université dans une société nouvelle 》, Relations 346 (février 1970), 55.57.

Fisher EA "Financial Accessibility to Higher Education in Canada in the 1960"s," Can. Assoc. University Teachers Bulletin XVIII, 4 (Summer, 1970), 92-106.

Gaudry, R. «L'Université et le développement au Canada 》, Association des Universites et Collèges du Canada, Délibérations 1970, 106-12. See also C.T. Bissell "Comments on the Theme Address, 113-14.

Macpherson, C.B "The University as Multiple Fool", C.A.U.T. Bull. XIX, 1 (Autumn, 1970) 3-7. Also in Mysterious East (February, 1971), 14-18.

Manley.Casimir, M. and I.E. Housego, "Equality of Educational Opportunity : 2 Canadian Perspective", Albetta Jour. Ed. Research, XVI, 2 (June, 1970), 79-88.

Pike, R.M. Who Doesn't Go to University and Why: a Study of Accessibility to Higher Education, Ottawa: A.U.C.C., 1970. Pp. 210.

Walmsley, N. Conadian Universities and International Development, Ottawa : A.U.C.C. 1970. Pp. 330.

Winthrop $\mathbf{H}_{\text {, }}$ "Are the Learned Professions Seceding from American Society ?" Dalbousie Rev. (Winter, 1970-71), 469-509.

B. University Government

Swingle, P.G. "Administrative Orbiting", C.A.U.T. Bull. XIX, 1 (Autumn 1970), 9.13. Watt, L.A.K. "The Choosing of a University President", OCUFA Newsletter III, 5 (Spring, 1970), 13-14.
C. University Finance

Atherton, P.J. "Financing Post-Secondary Education in Alberta", Alta. Jour. Ed. Research XVI, 3 (September, 1970), 137-48.

Hanly, C. et al. Who Pays: Universily Findancing in Ontario. Toronto: Lewis and Samuel, 1970. Pp. 168

Robinson, G. de B. "University Overhead Costs : a Critical Problem in Canada", Science Forum, III, 5 (October, 1970), 20-21.

E. University and the Economy

Atkinson, A.J., K.J. Barnes and E. Richardson. Canada's Higbly Qualified Manpower Resources, Ottawa: Dept. of Manpower \& Immigration, 1970. Pp. 304.

Carrier, H. "L'Université dans un monde à développer», Relations 354 (Novembre 1970), 306.09.

Riverin, A., L'Université et le développement socio-économique. Montreal : Publications Les Affaires, 1970 . Pp. 162

3. Curriculum and Teaching

C. Humanities

Lebel, M. "L'Enseignement universitaire de la littérature canadienne-française au Canada et à l'etranger \$, Culsure XXXI, 3 (septembre 1970), 238-44.

Dubuc, A. "L'Histoire au carrefour des sciences humaines", Revue d'Histoire de PAmerique Française XXIV, 3 (decembre 1970), 331-40.

Lamonde, Y. «L'Enseignement de la philosophie au Collège de Montréal 1790-1876 》, Culture XXXI 2 (juin 1970) 109.23; 3 (septembre 1970), 213-24; 4 (décembre $1970), 312.26$

D. Social Sciences

Gordon, S. Social Science and Modern Man, Toronto: U. of T. Press, 1970. Pp. 63. Verney, D.V. "The Education of a Political Scientist", Can. Journal Political Science III, 3 (September, 1970), 345-58.

Myers, C.R. "Journal Citations and Scientific Eminence in Contemporary Psychology", American Psychologist XXV (November, 1970), 1041-48.

Nelson, T.M. and W. Poley, "Publication Habits of Psychologists in Canadian Universities", Can. Psychologist XII, 1 (January, 1971), 68-76.

Morgan, J.G. "Contextual Factors in the Rise of Academic Sociology in the United States", Can. Rev. Soc. Anibrop. VII, 3 (August, 1970), 159-71.

E. Mathematics and the Sciences

Rogers, D., "Hostility to Science in the University : a Science Student View", Science Forum III, 4 (August, 1970), 3-6.

F. Professional Education

Koerte, A. "Architectural Education, Part I : the Global Village", Can Arcbisecture XV, 9 (September, 1970), 51-54; "Part II : Trends in Europe", 11 (November, 1970), 39-42. See also N. Pressman, 12 (December, 1970), 61-63.

Manning, P. "Architectural Education; the Wider View", Can. Arcbitect. XV, 6 (June, I970), 40-42. 
Gullett, D., A History of Dentistry in Canada. Toronto : U. of T. Press, 1971. Pp. 308. Lapp, P., J.W. Hodgins and C.B. MacKay., Ring of Iron : a Study of Engineering Education in Ontario, Toroato: Council of Ontario Universities, 1970. Pp. 154 Diemert N.E, and E.A. Holdaway, "Teacher Satisfaction in Team and Conventional Teaching Situations", Aliat. Jour. Ed. Research XVI, 2 (June, 1970), 111-12.

Elliott, A. "Predicting Success in Teaching", Alta. Jowr. Edut. Research, XVII, 2 (June, 1971), 69-76.

McLeish, J.B. "Student Attitudes to Teaching Methods", Alta. Journ. Ed. Research XVI, 3 (September, 1970), 179-88.

Mothersill, I. "Amalgamation of 'Teachers' College at Lakehead: a Case Study", OCUFA Newsletter III, 3 (January, 1970), 8-9.

Lucier, P., « Les Tâches de la théologie au Québec 》, Relations 346 (février 1970), 50-52. Isbelle, L., "Should Pupil Personnel Workers in Schools Have Both Teacher Training and Experience?"', Can. Counsellor IV, 3 (June, 1970), 165-73.

\section{Admissions}

Joly, J.M., "Le Service d'Admission au Collège et à l'Université (SACU) ", Prospec tives, VI, 3 (juin 1970), 150-53.

\section{J. Evaluation and Grading}

Fortier C a Conversion des cours actuels en un système de credits 》, Prospectives, VI, 5 (novembre 1970), 316-27.

Hone, A., J.P. Baillon et P.F. Caillibot, "Une Technique téaliste de l'évaluation de l'étudiant applicable aux grands groupes 》, Prospectives, VI, 2 (avtil 1970), 122-25.

K. Instructional Aids

Cropley, A.J. and P.F. Gross, "The Effectiveness of Computer-Assisted Instruction" Alta. Jour. Ed. Research XVI, 4 (December, 1970), 203-10.

4. Resedich and Scholarsbip

E. In Education

Aubin, G., « La Recherche en pédagogie ", Prospeatives VI, 1 (février 1970), 16-30.

5. Student and Student Services

\section{A. General}

Boucher, P., "L'Étudiant de 1985: une carrièe d'avenir ", Prospectives VI, 1 (février 1970), 46-54.

Danseren "P. "Finding New Ways to Communicate with Students", Science Fortum III, 5 (October, 1970), 24-26

Gagnon, J.A., "An Analysis of Student Life Developments in Quebec: Implications for Other Canadian Universities", Jour. Council. Univ. Student Services V, (Autumn-W inter, 1970), 7.21.

Lazure, J., "Les Jeunes Québécois encore à l'âge de la métaphysique 》, Prospectives, VI, 1 (février 1970), 31-45.

O'Brien, J.W., "Emerging Trends in Student Life : Implications for Student Services", Jour. Council. Univ. Student Services V, 2 (Autumn-Winter, 1970), 2-6.
Turcotte, R., «PBI sur le développement des services aux étudiants", Prospertives, VI, 4 (septembre 1970), 226-35.

B. Political and Social Development

Devall, W.B., "Support for Civil Liberties among English-Speaking Canadian University Students", Can. Jour. Polir. Science III, 3 (September, 1970), 433.49.

E. Counselling and Guidance

Romaniuk, E.W. and T.O. Maguire, "Computer-Assisted Guidance", Can. Counsellor IV, 3 (June, 1970), 149-60.

Talley, W.M., "Some Concerns about Group Experiences", Jour. Council. Untiv. Student Services, V, 2 (Autumn-Winter, 1970), 22-26

G. External Organizations

Hamilton, I., The Children's Crusade: the Story of the Company of Young Canadians, Toronto : Peter Martin, 1970. Pp. 312.

6. The Professor and Conditions of Work

C. Academic Freedom

Berland, A., "Academic Freedom and Tenure / Liberté Universitaire et permanence de l'emploi : Simon Fraser University Dispute", CAUT Bull. XIX, (Autumn, 1970), 65-85.

Canadian Association of University Teachers Committee on Academic Freedom and Tenure, "Academic Freedom and Tenure... Mount Allison University", CAUT Bull. XIX, 1 (Autumn, 1970), 51-58.

Monahan, E.J., "Academic Freedom and Tenure and C.A.U.T.: the First Twenty Years", CAUT Bull. XVIII, 4 (Summer, 1970), 80-91.

Polka, B., "Freedom and Responsibility in the University", OCUFA Newsletter III, 3 (January, 1970), 1-3.

D. Salary and Benefits

Bird, RM., "Tax Reform and the University Professor," OCUFA Newsletter, III, 3 (January, 1970), 4-5.

Canadian Association of University Teachers "Brief on Taxation", CAUT Bull. XIX, 1 (Autumn, 1970), 31-50.

Hanly, C.M.T. "Salary Action at the Provincial Level", OCUFA Newsletter III, 3 (January, 1970), 10-13; "Salary Policy", IV, I (September, 1970), 5-7.

\section{II. - NON-DEGREE GRANTING INSTITUTIONS}

6. Qneber

Belanger, G., «Réflexions sur l'implantation des CEGEP », Prospectives VI, 4 (septembre 1970), 262.76.

Boucher, R., "Le Collège-bibliothèque, une solution cadre ", Prospectives, VI, 5 (novembre 1970), 341.48. 
Gingras, P.-E., « $\boldsymbol{A}$ la Recherche des objectifs de l'enseignement collégial ", Prospertives, VI, 3 (juin 1970), 182-89.

"Rapport de la Commission d'Enquête sur la Communication au Collège de Maisonneuve ", Prospectives, VI, 5 (novembre 1970), 328.40.

Sauvé, J.-C., «Ia Regionalisation des CEGEP : comment utiliserons-nous cet outil ?", Prospectives, VI, 2 (avril 1970), 71-74.

Thibault, M., « Etudiants et professeurs : combien sont-ils dans les institutions privées et les CEGEP [en 1969-70] ? " Prospectives VI, I (février 1970), 8-15.

Thibault, M., \& Plus de 72,000 étudiants et 6,000 professeurs au niveau collégial pour l'annee 1970.71\%, Prospectives, VI, 6 (déecmbre 1970), 359-64. 